\title{
The regularity criterion for weak solutions to the $n$-dimensional Boussinesq system
}

\section{Xiaona Cui}

"Correspondence: cxn009@126.com College of Mathematics and Information Science, Henan Normal University, Xinxiang, 453007, P.R. China

\begin{abstract}
We consider the Boussinesq system in the homogeneous spaces of degree -1 . To narrow the gap for the existence of small regular solutions in $\dot{B}_{\infty, \infty}^{-1}\left(\mathbb{R}^{n}\right)$, the biggest homogeneous space of degree -1 among those embedded in the space of tempered distributions, we show small solutions in the homogeneous Besov space $\dot{B}_{p, \infty}^{-1+\frac{n}{p}}\left(\mathbb{R}^{n}\right)$, with $n \geq 2, n \leq p<\infty$.
\end{abstract}

MSC: $35 \mathrm{Q} 35 ; 76 \mathrm{~B} 03$

Keywords: Boussinesq equations; regularity; weak solution

\section{Introduction and main results}

The Cauchy problem of the Boussinesq system in $\mathbb{R}^{n}(n \geq 2)$ reads

$$
\left\{\begin{array}{l}
\partial_{t} u+u \cdot \nabla u-\mu \Delta u+\nabla \pi=\theta e_{n}, \quad(x, t) \in \mathbb{R}^{n} \times(0, \infty), \\
\partial_{t} \theta+u \cdot \nabla \theta-\kappa \Delta \theta=0, \\
\nabla \cdot u=0, \\
u(x, 0)=u_{0}, \quad \theta(x, 0)=\theta_{0},
\end{array}\right.
$$

where $u=u(x, t)$ and $\theta=\theta(x, t)$ denote the unknown velocity field and the scalar temperature in the content of thermal convection, respectively, and $\pi=\pi(x, t)$ the scalar density of the geophysical fluids, $\mu$ the constant kinematic viscosity, $\kappa>0$ the thermal diffusivity, and $e_{n}=(0,0, \ldots, 1)^{T}$. While $u_{0}, \theta_{0}$ are given initial data, with $\nabla \cdot u_{0}=0$ in the sense of distribution.

The Boussinesq system is extensively used in the atmospheric sciences and oceanographic turbulence (see [1-3] and references therein). The problem of the global regularity of the weak solutions of the 3D Boussinesq equations is a big open problem. It is meaningful to study the regularity of the weak solutions under additional critical growth conditions on the velocity or the pressure. Based on some analysis technique, there are some regularity criteria via the velocity of weak solutions in Besov spaces have been obtained in [4-6]. The pressure criterion is in [7-9]. By the velocity criterion, for the $n$-dimensional Boussinesq system, Yao et al. in [10] showed the local well-posedness and blow-up criteria in Besov-Morrey spaces $N_{p, q, r}^{s}\left(\mathbb{R}^{n}\right)$ in supercritical case $s>1+\frac{n}{p}, 1<q \leq p<\infty, 1 \leq r \leq \infty$, and critical case $s=1+\frac{n}{p}, 1<q \leq p<\infty, r=1$. Zhang et al. [11] got the existence of the

(c) The Author(s) 2017. This article is distributed under the terms of the Creative Commons Attribution 4.0 International License (http://creativecommons.org/licenses/by/4.0/), which permits unrestricted use, distribution, and reproduction in any medium, provided you give appropriate credit to the original author(s) and the source, provide a link to the Creative Commons license, and indicate if changes were made. 
2-dimensional inviscid Boussinesq equations in critical Besov spaces $B_{p, 1}^{\frac{2}{p}+1}\left(\mathbb{R}^{2}\right)$ and some blow-up criteria.

The global regularity of smooth solution of the 2D Boussinesq equations with the fractional dissipation has been researched recently in [12-18]. Several Beale-Kato-Majadatype regularity criterion have been obtained in [19-25]. There are also some results for the blow-up criteria for the Boussinesq equations (see $[26,27]$ and the references therein).

For the Navier-Stokes equations, Xin and Chen in [28] researched the small regular solutions in $\dot{B}_{\infty, \infty}^{-1}$, which is the biggest homogeneous space of degree -1 . The authors studied small solutions in the homogeneous Besov space $\dot{B}_{p, \infty}^{-1+\frac{n}{p}}\left(\mathbb{R}^{n}\right)$ and a homogeneous space defined by $\widehat{M}_{n}\left(\mathbb{R}^{n}\right)$. Here, motivated by the results in [28], our aim is to do some work addressing the small regular solutions of the $\mathrm{n}$-dimensional Boussinesq system in subcritical spaces $\dot{B}_{p, \infty}^{-1+\frac{n}{p}}\left(\mathbb{R}^{n}\right)$. The corresponding content in the space $\widehat{M}_{n}\left(\mathbb{R}^{n}\right)$ is of our further interest. This result partially extends the result in [28] in another system. More precisely, we will prove the following.

Theorem 1.1 Suppose $n \geq 2, n \leq p<\infty, 2-\frac{n}{p}<\alpha<2, u_{0} \in \dot{B}_{p, \infty}^{-1+\frac{n}{p}}\left(\mathbb{R}^{n}\right), \theta_{0} \in \dot{B}_{p, \infty}^{-1+\frac{n}{p}}\left(\mathbb{R}^{n}\right) \cap$ $\dot{B}_{p, \infty}^{-3+\frac{n}{p}}\left(\mathbb{R}^{n}\right)$ with $\operatorname{div} u_{0}=0$ in $\mathbb{R}^{n}$, and $\left\|u_{0}\right\|_{\dot{B}_{p, \infty}^{-1+\frac{n}{p}}}+\left\|\theta_{0}\right\|_{\dot{B}_{p, \infty}^{-1+\frac{n}{p}}}+\left\|\theta_{0}\right\|_{\dot{B}_{p, \infty}^{-3+\frac{n}{p}}} \leq \epsilon$ for some small constant $\epsilon=\epsilon(n, p, \alpha)$. Then system (1.1) admits a unique regular solution satisfying

$$
\begin{aligned}
& \|u(t)\|_{\dot{B}_{p, \infty}^{-1+\frac{n}{p}}}+t^{\frac{1}{2}}\|u(t)\|_{L_{\infty}}+t^{\frac{\alpha}{2}}\|u(t)\|_{\dot{B}_{p, \infty}^{\alpha-1+\frac{n}{p}}} \leq C\left(\left\|u_{0}\right\|_{\dot{B}_{p, \infty}^{-1+\frac{n}{p}}}+\left\|\theta_{0}\right\|_{\dot{B}_{p, \infty}^{-3+\frac{n}{p}}}\right), \\
& \|\theta(t)\|_{\dot{B}_{p, \infty}^{-1+\frac{n}{p}}}+t^{\frac{1}{2}}\|\theta(t)\|_{L_{\infty}}+t^{\frac{\alpha}{2}}\|\theta(t)\|_{\dot{B}_{p, \infty}^{\alpha-1+\frac{n}{p}}} \leq C\left\|\theta_{0}\right\|_{\dot{B}_{p, \infty}^{-1+\frac{n}{p}}} .
\end{aligned}
$$

This paper is structured as follows. In Section 2, we introduce the Besov spaces and the lemmas used later. In Section 3, we provide the proof of Theorem 1.1.

\section{Preliminary lemmas}

We denote (cf. [28])

$$
\left(e^{t \Delta} f\right)(x)=\int_{\mathbb{R}^{3}} h(x-y, t) f(y) d y
$$

with the heat kernel $h(x, t)=(4 \pi t)^{-n / 2} e^{-|x|^{2} /(4 t)}$. And the Fourier transform $\widehat{f}$ of $f \in \mathcal{S}$ is defined by

$$
\widehat{f}(\xi)=(2 \pi)^{-\frac{n}{2}} \int_{\mathbb{R}^{n}} f(x) e^{-i x \cdot \xi} d x
$$

Here $\mathcal{S}\left(\mathbb{R}^{n}\right)$ stands for the Schwartz class of rapidly decreasing smooth functions and $\mathcal{S}^{\prime}\left(\mathbb{R}^{n}\right)$ is the space of tempered distributions. The fractional order of the Laplacian is showed by the Fourier transform. For $\alpha \in \mathbb{R}$,

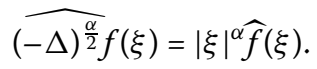

Due to the homogeneous counterpart Theorem 2.12.2 and the lifting property Theorem 5.2.3/1 in [29], the homogeneous Besov spaces can be given as follows. 
Definition 2.1 For $1 \leq p, q \leq \infty$ and $-\infty<\alpha<\infty$, the homogeneous Besov spaces are defined

$$
\dot{B}_{p, q}^{\alpha}\left(\mathbb{R}^{n}\right)=\left\{u \in \mathcal{S}^{\prime}\left(\mathbb{R}^{n}\right):\|u\|_{\dot{B}_{p, q}^{\alpha}}<\infty\right\}
$$

where

$$
\|u\|_{\dot{B}_{p, q}^{\alpha}}= \begin{cases}\left(\int_{0}^{\infty} t^{(1-\alpha / 2) q}\left\|\Delta e^{t \Delta} u\right\|_{L^{p}}^{q} \frac{d t}{t}\right)^{1 / q}, & \text { for } 0<\alpha<2(q \neq \infty), \\ \sup _{t>0} t^{1-\alpha / 2}\left\|\Delta e^{t \Delta} u\right\|_{L^{p}}, & \text { for } 0<\alpha<2(q=\infty), \\ \left\|(-\Delta)^{(\alpha-1) / 2} u\right\|_{\dot{B}_{p, q}^{1},} & \text { for others. }\end{cases}
$$

An important property of the homogeneous spaces is its invariance under the following space scaling:

$$
\|u(\lambda \cdot)\|_{\dot{B}_{p, q}^{\alpha}}=\lambda^{\alpha-n / p}\|u(\cdot)\|_{\dot{B}_{p, q}^{\alpha}} \cdot
$$

And furthermore, if $s<0$, the homogeneous Besov spaces $\dot{B}_{p, q}^{s}\left(\mathbb{R}^{n}\right)$ can be equivalently defined as follows (cf. [30]).

Lemma 2.1 Suppose $1 \leq p, q \leq \infty, s<0$. Then $f \in \dot{B}_{p, q}^{s}\left(\mathbb{R}^{n}\right)$ if and only if

$$
\begin{aligned}
& \left(\int_{0}^{\infty}\left(t^{-\frac{s}{2}}\left\|e^{t \Delta} f\right\|_{L^{p}\left(\mathbb{R}^{n}\right)}\right)^{q} \frac{d t}{t}\right)^{\frac{1}{q}}<\infty, \quad 1 \leq q<\infty, \\
& \sup _{t>0} t^{-\frac{s}{2}}\left\|e^{t \Delta} f\right\|_{L^{p}\left(\mathbb{R}^{n)}\right.}<\infty, \quad q=\infty .
\end{aligned}
$$

In particular, for the degree of -1 , we have

$$
\dot{B}_{\infty, \infty}^{-1}\left(\mathbb{R}^{n}\right)=\left\{f \in \mathcal{S}^{\prime}\left(\mathbb{R}^{n}\right):\|f\|_{\dot{B}_{\infty, \infty}^{-1}}=\sup _{t>0} t^{\frac{1}{2}}\left\|e^{t \Delta} f\right\|_{L^{\infty}}<\infty\right\} .
$$

It is well known that $\dot{B}_{\infty, \infty}^{-1}\left(\mathbb{R}^{n}\right)$ is the biggest critical homogeneous space of degree -1 , and as shown by Frazier, Jaweth and Weiss [31], any critical homogeneous space continuously embedded in $\mathcal{S}^{\prime}\left(\mathbb{R}^{n}\right)$ is also continuously embedded into $\dot{B}_{\infty, \infty}^{-1}\left(\mathbb{R}^{n}\right)$.

Next we introduce the interpolation theorem in $[29,32]$.

Lemma 2.2 Suppose $0<\theta<1,1 \leq p, q \leq \infty,-\infty<\alpha<\beta<\infty$, and

$$
\gamma=(1-\theta) \alpha+\theta \beta
$$

Then

$$
\left(\dot{B}_{p, \infty}^{\alpha}\left(\mathbb{R}^{n}\right), \dot{B}_{p, \infty}^{\beta}\left(\mathbb{R}^{n}\right)\right)_{\theta, q}=\dot{B}_{p, q}^{\gamma}\left(\mathbb{R}^{n}\right),
$$

where $(\cdot, \cdot)_{\theta, q}$ denotes the real interpolation functor. 
We express $(1.1)_{1,2}$ in the integral form as

$$
\begin{aligned}
& u(t)=e^{t \Delta} \mathbb{P} u_{0}-\int_{0}^{t} e^{(t-s) \Delta} \mathbb{P} \nabla \cdot(u(s) \otimes u(s)) d s+\int_{0}^{t} e^{(t-s) \Delta} \mathbb{P} \theta e_{n} d s, \\
& \theta(t)=e^{t \Delta} \theta_{0}-\int_{0}^{t} e^{(t-s) \Delta} \nabla \cdot(u(s) \otimes \theta(s)) d s,
\end{aligned}
$$

where $\mathbb{P}$ is the Helmholtz-Weyl projection onto a divergence free vector fields defined by

$$
\mathbb{P}=\left\{P_{j, k}\right\}_{j, k=1, \ldots, n}=\left\{\delta_{j, k}+R_{j} R_{k}\right\}_{j, k=1, \ldots, n}
$$

here $\delta_{j, k}$ is the Kronecker symbol and $R_{j}=\partial_{j}(-\Delta)^{-\frac{1}{2}}$ are the Riesz transforms. To prove the existence of the regular solution in $L^{\infty}\left((0, \infty), L^{n}\left(\mathbb{R}^{n}\right)\right)$, we need the $L^{p}-L^{q}$ type estimate for $e^{t \Delta}$ in Lebesgue spaces and Besov spaces. See [30] for the proof of the following lemma.

Lemma 2.3 Suppose $1 \leq p, q \leq \infty$. Thus, the following estimates hold:

$$
\begin{aligned}
& \left\|e^{t \Delta} f\right\|_{L^{p}\left(\mathbb{R}^{n)}\right.} \leq C\|f\|_{L^{p}\left(\mathbb{R}^{n}\right)}, \\
& \left\|\mathbb{P} \nabla e^{t \Delta} f\right\|_{L^{p}\left(\mathbb{R}^{n}\right)} \leq C t^{-\frac{1}{2}}\|f\|_{L^{p}\left(\mathbb{R}^{n}\right)}, \\
& \left\|e^{t \Delta} f\right\|_{\dot{B}_{p, q}^{s_{1}\left(\mathbb{R}^{n}\right)}} \leq C t^{-\frac{s_{1}-s_{2}}{2}}\|f\|_{\dot{B}_{p, q}^{s_{2}}\left(\mathbb{R}^{n}\right)}, \quad s_{2} \leq s_{1} .
\end{aligned}
$$

If $s_{1}, s_{2}<\frac{n}{p}$ and $s_{1}+s_{2}+n \min \left\{0,1-\frac{2}{p}\right\}>0$, then, for a positive constant $C$, we have

$$
\|u v\|_{\dot{B}_{p, q}^{s_{1}+s_{2}-\frac{n}{p}}} \leq C\|u\|_{\dot{B}_{p, q}^{s_{1}}}\|u\|_{\dot{B}_{p, q}^{s_{2}}}
$$

Due to the linearization of the Boussinesq system (1.1), we consider a priori estimates for the Stokes equations. Chen and Xin in [28] gave the estimates in homogeneous spaces. The Stokes equations in $\mathbb{R}^{n}, n \geq 2$ read

$$
\left\{\begin{array}{l}
\partial_{t} u-\Delta u+\nabla \pi=\nabla \cdot f \quad\left(f=\left(f_{i j}(x, t)\right)_{n \times n}\right), \\
\nabla \cdot u=0 \\
u(0)=a .
\end{array}\right.
$$

We now state the main estimate about the Stokes equations in homogeneous spaces of Chen and Xin in [28], which will be used later.

Lemma 2.4 Let $0<\alpha<2,2 \leq n \leq p \leq \infty, 1 \leq q \leq \infty$, and

$$
a \in \mathcal{S}^{\prime}\left(\mathbb{R}^{n}\right), \quad f(t) \in \dot{B}_{p, \infty}^{\alpha-2+\frac{n}{p}}\left(\mathbb{R}^{n}\right) .
$$

Then the solution of equation (2.1) in the following integral formulation:

$$
u(t)=e^{t \Delta} \mathbb{P} a+\int_{0}^{t} e^{(t-s) \Delta} \mathbb{P} \nabla \cdot f(s) d s
$$


satisfies the estimates

$$
\begin{aligned}
& \|u(t)\|_{\dot{B}_{p, q}^{-1+\frac{n}{p}}} \leq C\|a\|_{\dot{B}_{p, q}^{-1+\frac{n}{p}}}+C \sup _{0<s<t} s^{\frac{\alpha}{2}}\|f(s)\|_{\dot{B}_{p, \infty}^{\alpha-2+\frac{n}{p}}}, \\
& t^{\frac{\alpha}{2}}\|u(t)\|_{\dot{B}_{p, \infty}^{\alpha-1+\frac{n}{p}}} \leq C\|a\|_{\dot{B}_{p, \infty}^{-1+\frac{n}{p}}}+C \sup _{0<s<t} s^{\frac{\alpha}{2}}\|f(s)\|_{\dot{B}_{p, \infty}^{\alpha-2+\frac{n}{p}}},
\end{aligned}
$$

provided that the right-hand sides of the above inequalities are finite, respectively.

Similar to Lemma 2.4, we have the analogous results.

Lemma 2.5 Under the same conditions with $\alpha, p, q$ in Lemma 2.4, and $f \in \dot{B}_{p, \infty}^{\alpha-3+\frac{n}{p}}$, the following integral equation:

$$
u(t)=e^{t \Delta} \mathbb{P} a+\int_{0}^{t} e^{(t-s) \Delta} \mathbb{P} f(s) d s
$$

satisfies the estimation

$$
\begin{aligned}
& \|u(t)\|_{\dot{B}_{p, q}^{-1+\frac{n}{p}}} \leq C\|a\|_{\dot{B}_{p, q}^{-1+\frac{n}{p}}}+C \sup _{0<s<t} s^{\frac{\alpha}{2}}\|f(s)\|_{\dot{B}_{p, \infty}^{\alpha-3+\frac{n}{p}}}, \\
& t^{\frac{\alpha}{2}}\|u(t)\|_{\dot{B}_{p, \infty}^{\alpha-1+\frac{n}{p}}} \leq C\|a\|_{\dot{B}_{p, \infty}^{-1+\frac{n}{p}}}+C \sup _{0<s<t} s^{\frac{\alpha}{2}}\|f(s)\|_{\dot{B}_{p, \infty}^{\alpha-3+\frac{n}{p}}} .
\end{aligned}
$$

Proof By Definition 2.1, we estimate the term $\left\|\int_{0}^{t} e^{(t-s) \Delta} \mathbb{P} f(s) d s\right\|_{\dot{B}_{p, \infty}^{\alpha-1+\frac{n}{p}}\left(\mathbb{R}^{n}\right)}$ as follows:

$$
\begin{aligned}
& \left\|\int_{0}^{t} e^{(t-s) \Delta} \mathbb{P} f(s) d s\right\|_{\dot{B}_{p, \infty}^{\alpha-1+\frac{n}{p}}\left(\mathbb{R}^{n}\right)} \\
& =\sup _{\tau>0} \tau^{1-\left(\alpha-1+\frac{n}{p}\right) / 2}\left\|\Delta e^{\tau \Delta} \int_{0}^{t} e^{(t-s) \Delta} \mathbb{P} f(s) d s\right\|_{\dot{B}_{p, \infty}^{\alpha-1+\frac{n}{p}}\left(\mathbb{R}^{n}\right)} \\
& \leq C \sup _{\tau>0} \tau^{1-\left(\alpha-1+\frac{n}{p}\right) / 2} \int_{0}^{t}\left\|\Delta e^{(\tau+t-s) \Delta} \mathbb{P} f(s)\right\|_{L^{p}\left(\mathbb{R}^{n}\right)} d s \\
& \leq C \sup _{\tau>0} \tau^{1-\left(\alpha-1+\frac{n}{p}\right) / 2} \int_{0}^{t}(\tau+t-s)^{-2+\left(\alpha-1+\frac{n}{p}\right) / 2}\|f(s)\|_{\dot{B}_{p, \infty}^{\alpha-3+\frac{n}{p}}\left(\mathbb{R}^{n}\right)} d s \\
& \leq C \sup _{\tau>0} \tau^{1-\left(\alpha-1+\frac{n}{p}\right) / 2} \int_{0}^{t}(\tau+t-s)^{-2+\left(\alpha-1+\frac{n}{p}\right) / 2} s^{-\frac{\alpha}{2}} d s \sup _{0<s<t} s^{\frac{\alpha}{2}}\|f(s)\|_{\dot{B}_{p, \infty}^{\alpha-3+\frac{n}{p}}\left(\mathbb{R}^{n}\right)} \\
& =C \sup _{\tau>0} \tau^{1-\left(\alpha-1+\frac{n}{p}\right) / 2}\left(\int_{0}^{\frac{t}{2}}+\int_{\frac{t}{2}}^{t}\right)(\tau+t-s)^{-2+\left(\alpha-1+\frac{n}{p}\right) / 2} s^{-\frac{\alpha}{2}} d s \\
& \quad \times \sup _{0<s<t} s^{\frac{\alpha}{2}}\|f(s)\|_{\dot{B}_{p, \infty}^{\alpha-3+\frac{n}{p}}\left(\mathbb{R}^{n}\right)} .
\end{aligned}
$$

For the term $I=\int_{0}^{\frac{t}{2}}(\tau+t-s)^{-2+\left(\alpha-1+\frac{n}{p}\right) / 2} s^{-\frac{\alpha}{2}} d s$, due to $0<s<\frac{t}{2}$, thus

$$
\tau+t-s>\frac{t}{2}+\tau=\frac{1}{2}(t+2 \tau)>\frac{1}{2}(t+\tau)
$$


Gui Boundary Value Problems ( 2017) 2017:44

Page 6 of 12

and the fact that $-2+\left(\alpha-1+\frac{n}{p}\right) / 2<0$, we have

$$
\begin{aligned}
I & \leq\left(\frac{1}{2}\right)^{-2+\left(\alpha-1+\frac{n}{p}\right) / 2} \int_{0}^{\frac{t}{2}}(t+\tau)^{-2+\left(\alpha-1+\frac{n}{p}\right) / 2} s^{-\frac{\alpha}{2}} d s \\
& =\left(\frac{1}{2}\right)^{-2+\left(\alpha-1+\frac{n}{p}\right) / 2}(t+\tau)^{-2+\left(\alpha-1+\frac{n}{p}\right) / 2} \int_{0}^{\frac{t}{2}} s^{-\frac{\alpha}{2}} d s \\
& \leq C(t+\tau)^{-2+\left(\alpha-1+\frac{n}{p}\right) / 2} \int_{0}^{t} s^{-\frac{\alpha}{2}} d s .
\end{aligned}
$$

As regards the term $I I=\int_{\frac{t}{2}}^{t}(\tau+t-s)^{-2+\left(\alpha-1+\frac{n}{p}\right) / 2} s^{-\frac{\alpha}{2}} d s$, since $\frac{t}{2}<s<t$,

$$
s^{-\frac{\alpha}{2}}<\left(\frac{1}{2}\right)^{-\frac{\alpha}{2}} t^{-\frac{\alpha}{2}}
$$

therefore

$$
I I \leq\left(\frac{1}{2}\right)^{-\frac{\alpha}{2}} t^{-\frac{\alpha}{2}} \int_{0}^{t}(\tau+t-s)^{-2+\left(\alpha-1+\frac{n}{p}\right) / 2} d s
$$

Thereby, the estimate becomes

$$
\begin{aligned}
& \left\|\int_{0}^{t} e^{(t-s) \Delta} \mathbb{P} f(s) d s\right\|_{\dot{B}_{p, \infty}^{\alpha-1+\frac{n}{p}}\left(\mathbb{R}^{n}\right)} \\
& \quad \leq \sup _{\tau>0} \tau^{1-\left(\alpha-1+\frac{n}{p}\right) / 2}(t+\tau)^{-2+\left(\alpha-1+\frac{n}{p}\right) / 2} \int_{0}^{t} s^{-\frac{\alpha}{2}} d s \sup _{0<s<t} s^{\frac{\alpha}{2}}\|f(s)\|_{\dot{B}_{p, \infty}^{\alpha-3+\frac{n}{p}}} \\
& \quad+\sup _{\tau>0} \tau^{1-\left(\alpha-1+\frac{n}{p}\right) / 2} t^{-\frac{\alpha}{2}} \int_{0}^{t}(t+\tau-s)^{-2+\left(\alpha-1+\frac{n}{p}\right) / 2} d s \sup _{0<s<t} s^{\frac{\alpha}{2}}\|f(s)\|_{\dot{B}_{p, \infty}^{\alpha-3+\frac{n}{p}}} \\
& =I I I+I V .
\end{aligned}
$$

Due to the definite integral $\int_{0}^{t} s^{-\frac{\alpha}{2}} d s=\frac{2}{2-\alpha} t^{1-\frac{\alpha}{2}}$, we obtain

$$
\begin{aligned}
I I I & \leq C \sup _{\tau>0}\left(\frac{\tau}{t+\tau}\right)^{1-\left(\alpha-1+\frac{n}{p}\right) / 2} \frac{t}{t+\tau} \cdot t^{-\frac{\alpha}{2}} \sup _{0<s<t} s^{\frac{\alpha}{2}}\|f(s)\|_{\dot{B}_{p, \infty}^{\alpha-3+\frac{n}{p}}} \\
& \leq C t^{-\frac{\alpha}{2}} \sup _{0<s<t} s^{\frac{\alpha}{2}}\|f(s)\|_{\dot{B}_{p, \infty}^{\alpha-3+\frac{n}{p}} .}
\end{aligned}
$$

According to the fact that

$$
\begin{aligned}
\int_{0}^{t}(\tau+t-s)^{-2+\left(\alpha-1+\frac{n}{p}\right) / 2} d s & =\int_{0}^{t}\left(\tau+s^{\prime}\right)^{-2+\left(\alpha-1+\frac{n}{p}\right) / 2} d s^{\prime} \\
& =\tau^{2-\left(\alpha-1+\frac{n}{p}\right) / 2} \int_{0}^{t}\left(1+\frac{s}{\tau}\right)^{-2+\left(\alpha-1+\frac{n}{p}\right) / 2} d s \\
& =\tau^{2-\left(\alpha-1+\frac{n}{p}\right) / 2} \int_{0}^{\frac{t}{\tau}}(1+s)^{-2+\left(\alpha-1+\frac{n}{p}\right) / 2} \tau d s
\end{aligned}
$$


thus

$$
\begin{aligned}
I V \leq & C \sup _{\tau>0} \tau^{1-\left(\alpha-1+\frac{n}{p}\right) / 2} t^{-\frac{\alpha}{2}} \tau^{-2+\left(\alpha-1+\frac{n}{p}\right) / 2} \tau \int_{0}^{\frac{t}{\tau}}(1+s)^{-2+\left(\alpha-1+\frac{n}{p}\right) / 2} d s \\
& \times \sup _{0<s<t} s^{\frac{\alpha}{2}}\|f(s)\|_{\dot{B}_{p, \infty}^{\alpha-3+\frac{n}{p}}} \\
= & t^{-\frac{\alpha}{2}} \sup _{\tau>0} \int_{0}^{\frac{t}{\tau}}(1+s)^{-2+\left(\alpha-1+\frac{n}{p}\right) / 2} d s \sup _{0<s<t} s^{\frac{\alpha}{2}}\|f(s)\|_{\dot{B}_{p, \infty}^{\alpha-3+\frac{n}{p}} .}
\end{aligned}
$$

Finally, we get

$$
\left\|\int_{0}^{t} e^{(t-s) \Delta} \mathbb{P} f(s) d s\right\|_{\dot{B}_{p, \infty}^{\alpha-1+\frac{n}{p}}\left(\mathbb{R}^{n}\right)} \leq C t^{-\frac{\alpha}{2}} \sup _{0<s<t} s^{\frac{\alpha}{2}}\|f(s)\|_{\dot{B}_{p, \infty}^{\alpha-3+\frac{n}{p}}} .
$$

For the estimate of the initial term $\left\|e^{t \Delta} \mathbb{P} a\right\|_{\dot{B}_{p, \infty}^{\alpha-1+\frac{n}{p}}}$, we have

$$
\begin{aligned}
\left\|e^{t \Delta} \mathbb{P} a\right\|_{\dot{B}_{p, \infty}^{\alpha-1+\frac{n}{p}}} & =\sup _{\tau>0} \tau^{1-\left(\alpha-1+\frac{n}{p}\right) / 2}\left\|\Delta e^{\tau \Delta} e^{t \Delta} \mathbb{P} a\right\|_{L^{p}} \\
& =\sup _{\tau>0} \tau^{1-\left(\alpha-1+\frac{n}{p}\right) / 2}\left\|\Delta e^{(t+\tau) \Delta} \mathbb{P} a\right\|_{L^{p}} \\
& \leq C \sup _{\tau>0} \tau^{1-\left(\alpha-1+\frac{n}{p}\right) / 2}(t+\tau)^{-1+\left(\alpha-1+\frac{n}{p}\right) / 2}(t+\tau)^{-\frac{\alpha}{2}}\|a\|_{\dot{B}_{p, \infty}^{-1+\frac{n}{p}}} \\
& \leq C \sup _{\tau>0} \tau^{1-\left(\alpha-1+\frac{n}{p}\right) / 2}(t+\tau)^{-1+\left(\alpha-1+\frac{n}{p}\right) / 2} t^{-\frac{\alpha}{2}}\|a\|_{\dot{B}_{p, \infty}^{-1+\frac{n}{p}}}
\end{aligned}
$$

because of the inequality

$$
\sup _{\tau>0} \tau^{1-\left(\alpha-1+\frac{n}{p}\right) / 2}(t+\tau)^{-1+\left(\alpha-1+\frac{n}{p}\right) / 2}<1,
$$

then we obtain

$$
\left\|e^{t \Delta} \mathbb{P} a\right\|_{\dot{B}_{p, \infty}^{\alpha-1+\frac{n}{p}}} \leq C t^{-\frac{\alpha}{2}}\|a\|_{\dot{B}_{p, \infty}^{-1+\frac{n}{p}}} .
$$

Equations (2.3) and (2.4) imply the second inequality of the lemma. Now we turn to the first inequality. By a similar method, we have

$$
\begin{aligned}
\left\|u(t)-e^{t \Delta} \mathbb{P} a\right\|_{\dot{B}_{p, \infty}^{-1+\frac{n}{p}}} & \leq C \int_{0}^{t}\left\|e^{(t-s) \Delta} f(s)\right\|_{\dot{B}_{p, \infty}^{-1+\frac{n}{p}}} d s \\
& \leq C \int_{0}^{t}(t-s)^{-1+\frac{\alpha}{2}}\|f(s)\|_{\dot{B}_{p, \infty}^{\alpha-3+\frac{n}{p}}} d s \\
& \leq C \int_{0}^{t}(t-s)^{-1+\frac{\alpha}{2}} s^{-\frac{\alpha}{2}} d s \sup _{0<s<t} s^{\frac{\alpha}{2}}\|f(s)\|_{\dot{B}_{p, \infty}^{\alpha-3+\frac{n}{p}}} \\
& \leq C \sup _{0<s<t} s^{\frac{\alpha}{2}}\|f(s)\|_{\dot{B}_{p, \infty}^{\alpha-3+\frac{n}{p}}} .
\end{aligned}
$$

On the other hand, by Lemma 2.3, we get

$$
\left\|e^{t \Delta} \mathbb{P} a\right\|_{\dot{B}_{p, \infty}^{-1+\frac{n}{p}}} \leq C\|a\|_{\dot{B}_{p, \infty}^{-1+\frac{n}{p}}} .
$$

Therefore, the expressions (2.5) and (2.6) verify the first inequality of the lemma. 
Lemma 2.6 Suppose $2-\frac{n}{p}<\alpha<2,2 \leq n \leq p \leq \infty$, and

$$
a \in \dot{B}_{p, \infty}^{-3+\frac{n}{p}}, \quad f \in \dot{B}_{p, \infty}^{\alpha-4+\frac{n}{p}}
$$

Thus equation (2.2) satisfies the estimate

$$
t^{\frac{\alpha}{2}}\|u(t)\|_{\dot{B}_{p, \infty}^{\alpha-3+\frac{n}{p}}} \leq C\|a\|_{\dot{B}_{p, \infty}^{-3+\frac{n}{p}}}+C \sup _{0<s<t} s^{\frac{\alpha}{2}}\|f(s)\|_{\substack{\dot{B}_{p, \infty}-4+\frac{n}{p} \\ \text {. }}} .
$$

Proof By the definition in Lemma 2.1 and Lemma 2.3, we have

$$
\begin{aligned}
& \left\|\int_{0}^{t} e^{(t-s) \Delta} \nabla f d s\right\|_{\dot{B}_{p, \infty}^{\alpha-3+\frac{n}{p}}} \\
& \quad \leq \sup _{\tau>0} \tau^{-\frac{\alpha-3+\frac{n}{p}}{2}} \int_{0}^{t}\left\|e^{(t+\tau-s) \Delta} \nabla f\right\|_{L^{p}} d s \\
& \quad \leq \sup _{\tau>0} \tau^{-\frac{\alpha-3+\frac{n}{p}}{2}} \int_{0}^{t}(t+\tau-s)^{-\frac{1}{2}+\frac{\alpha-4+\frac{n}{p}}{2}}(t+\tau-s)^{-\frac{\alpha-4+\frac{n}{p}}{2}}\left\|e^{(t+\tau-s) \Delta} \nabla f\right\|_{L^{p}} d s \\
& \quad \leq \sup _{\tau>0} \tau^{-\frac{\alpha-3+\frac{n}{p}}{2}} \int_{0}^{t}(t+\tau-s)^{\frac{\alpha-5+\frac{n}{p}}{2}} s^{-\frac{\alpha}{2}} d s \sup _{s>0} s^{\frac{\alpha}{2}}\|f\|_{\dot{B}_{p, \infty}^{\alpha-4+\frac{n}{p}}},
\end{aligned}
$$

the way to deal with the above estimate is similar to the one in Lemma 2.4, we omit it here. Finally, we get

$$
\left\|\int_{0}^{t} e^{(t-s) \Delta} \nabla f d s\right\|_{\dot{B}_{p, \infty}^{\alpha-3+\frac{n}{p}}} \leq C t^{-\frac{\alpha}{2}} \sup _{s>0} s^{\frac{\alpha}{2}}\|f\|_{\dot{B}_{p, \infty}^{\alpha-4+\frac{n}{p}} .} .
$$

For the initial term, according to Lemma 2.3, we obtain

$$
\left\|e^{t \Delta} \theta_{0}\right\|_{\dot{B}_{p, \infty}^{\alpha-3+\frac{n}{p}}} \leq C t^{-\frac{\alpha}{2}}\left\|\theta_{0}\right\|_{\dot{B}_{p, \infty}^{-3+\frac{n}{p}}} .
$$

Therefore, we get the proof.

\section{The proof of Theorem 1.1}

This section is devoted to the proof of Theorem 1.1. For simplicity, without loss of generality, we assume $\mu=\kappa=1$. In order to deal with the convection terms, we observe the interesting interpolation results

$$
\begin{aligned}
& \left(\dot{B}_{p, \infty}^{-1+\frac{n}{p}}\left(\mathbb{R}^{n}\right), \dot{B}_{p, \infty}^{\alpha-1+\frac{n}{p}}\left(\mathbb{R}^{n}\right)\right)_{\frac{1}{\alpha}, 1}=\dot{B}_{p, 1}^{\frac{n}{p}}\left(\mathbb{R}^{n}\right), \\
& \left(\dot{B}_{p, \infty}^{-1+\frac{n}{p}}\left(\mathbb{R}^{n}\right), \dot{B}_{p, \infty}^{\alpha-1+\frac{n}{p}}\left(\mathbb{R}^{n}\right)\right)_{1-\frac{1}{\alpha}, \infty}=\dot{B}_{p, \infty}^{\alpha-2+\frac{n}{p}}\left(\mathbb{R}^{n}\right) .
\end{aligned}
$$

It is well known that, for $s>0, \dot{B}_{p, q}^{s}\left(\mathbb{R}^{n}\right) \cap L^{\infty}\left(\mathbb{R}^{n}\right)$ is an algebra. Moreover, there exists the following embedding relations $(c f .[28,29])$ :

$$
\dot{B}_{p, 1}^{\frac{n}{p}}\left(\mathbb{R}^{n}\right) \hookrightarrow \dot{B}_{\infty, 1}^{0}\left(\mathbb{R}^{n}\right) \hookrightarrow L^{\infty}\left(\mathbb{R}^{n}\right) \hookrightarrow \dot{B}_{\infty, \infty}^{0}\left(\mathbb{R}^{n}\right)
$$


Thus by the embedding and interpolation results, we have

$$
\|u\|_{L^{\infty}} \leq\|u\|_{\dot{B}_{\infty, 1}^{0}} \leq\|u\|_{\dot{B}_{p, 1}^{\frac{n}{p}}} \leq C\|u\|_{\dot{B}_{p, \infty}^{-1+\frac{n}{p}}}^{1-\frac{1}{\alpha}}\|u\|_{\dot{B}_{p, \infty}^{\alpha-1+\frac{n}{p}}}^{\frac{1}{\alpha}}
$$

and

$$
\|u\|_{\dot{B}_{p, \infty}^{\alpha-2+\frac{n}{p}}} \leq C\|u\|_{\dot{B}_{p, \infty}^{-1+\frac{n}{p}}}^{\frac{1}{\alpha}}\|u\|_{\dot{B}_{p, \infty}^{\alpha-1+\frac{n}{p}}}^{1-\frac{1}{\alpha}} .
$$

And then due to the inequality $a^{1-\beta} b^{\beta} \leq a+b, 0<\beta<1, a, b>0$, we have

$$
\left\|u\left(t^{\frac{1}{2}} \cdot, t\right)\right\|_{L^{\infty}}+\left\|u\left(t^{\frac{1}{2}} \cdot, t\right)\right\|_{\dot{B}_{p, \infty}^{\alpha-2+\frac{n}{p}}} \leq C\left(\left\|u\left(t^{\frac{1}{2}} \cdot, t\right)\right\|_{\dot{B}_{p, \infty}^{-1+\frac{n}{p}}}+\left\|u\left(t^{\frac{1}{2}} \cdot, t\right)\right\|_{\dot{B}_{p, \infty}^{\alpha-1+\frac{n}{p}}}\right) .
$$

By a variable transformation, (3.1) implies

$$
t^{\frac{1}{2}}\|u(t)\|_{L^{\infty}}+t^{\frac{\alpha-1}{2}}\|u(t)\|_{\dot{B}_{p, \infty}^{\alpha-2+\frac{n}{p}}} \leq C\left(\|u(t)\|_{\dot{B}_{p, \infty}^{-1+\frac{n}{p}}}+t^{\frac{\alpha}{2}}\|u(t)\|_{\dot{B}_{p, \infty}^{\alpha-1+\frac{n}{p}}}\right) .
$$

Equation (3.2) means

$$
t^{\frac{1}{2}}\|u(t)\|_{L^{\infty}} \leq C\left(\|u(t)\|_{\dot{B}_{p, \infty}^{-1+\frac{n}{p}}}+t^{\frac{\alpha}{2}}\|u(t)\|_{\dot{B}_{p, \infty}^{\alpha-1+\frac{n}{p}}}\right) .
$$

Thus to prove the two equations in Theorem 1.1, we only need to show that

$$
\begin{aligned}
& \|u(t)\|_{\dot{B}_{p, \infty}^{-1+\frac{n}{p}}}+t^{\frac{\alpha}{2}}\|u(t)\|_{\dot{B}_{p, \infty}^{\alpha-1+\frac{n}{p}}} \leq C\left(\left\|u_{0}\right\|_{\dot{B}_{p, \infty}^{-1+\frac{n}{p}}}+\left\|\theta_{0}\right\|_{\dot{B}_{p, \infty}^{-3+\frac{n}{p}}}\right), \\
& \|\theta(t)\|_{\dot{B}_{p, \infty}^{-1+\frac{n}{p}}}+t^{\frac{\alpha}{2}}\|\theta(t)\|_{\dot{B}_{p, \infty}^{\alpha-1+\frac{n}{p}}} \leq C\left\|\theta_{0}\right\|_{\dot{B}_{p, \infty}^{-1+\frac{n}{p}}} .
\end{aligned}
$$

Set

$$
\begin{aligned}
& U=\left\{u \in L^{\infty}\left(0, \infty ; \dot{B}_{p, \infty}^{-1+\frac{n}{p}}\left(\mathbb{R}^{n}\right)\right) \mid \nabla \cdot u=0,\|u\|_{U}<\infty\right\} \\
& \Theta=\left\{\theta \in L^{\infty}\left(0, \infty ; \dot{B}_{p, \infty}^{-1+\frac{n}{p}}\left(\mathbb{R}^{n}\right)\right) \mid\|\theta\|_{\Theta}<\infty\right\}
\end{aligned}
$$

with

$$
\begin{aligned}
& \|u\|_{U}=\sup _{t>0}\left(\|u(t)\|_{\dot{B}_{p, \infty}^{-1+\frac{n}{p}}}+t^{\frac{\alpha}{2}}\|u(t)\|_{\dot{B}_{p, \infty}^{\alpha-1+\frac{n}{p}}}\right), \\
& \|\theta\|_{\Theta}=\sup _{t>0}\left(\|\theta(t)\|_{\dot{B}_{p, \infty}^{-1+\frac{n}{p}}}+t^{\frac{\alpha}{2}}\|\theta(t)\|_{\dot{B}_{p, \infty}^{\alpha-1+\frac{n}{p}}}\right),
\end{aligned}
$$

and

$$
\begin{aligned}
& M u(t)=e^{t \Delta} u_{0}-\int_{0}^{t} e^{(t-s) \Delta} \mathbb{P} \nabla \cdot(u(s) \otimes u(s)) d s+\int_{0}^{t} e^{(t-s) \Delta} \mathbb{P} \theta e_{n} d s, \\
& \bar{M} \theta(t)=e^{t \Delta} \theta_{0}-\int_{0}^{t} e^{(t-s) \Delta} \nabla \cdot(u(s) \otimes \theta(s)) d s .
\end{aligned}
$$


We will show $M$ and $\bar{M}$ are the contraction operators mapping a ball of $U$ into itself and a ball of $\Theta$ into itself, respectively. Now we deal with the operator $\bar{M}$. Observing that (3.1) and (3.2) are also true for $\theta$, we have

$$
t^{\frac{\alpha-1}{2}}\|\theta(t)\|_{\dot{B}_{p, \infty}^{\alpha-2+\frac{n}{p}}} \leq C\left(\|\theta(t)\|_{\dot{B}_{p, \infty}^{-1+\frac{n}{p}}}+t^{\frac{\alpha}{2}}\|u(t)\|_{\dot{B}_{p, \infty}^{\alpha-1+\frac{n}{p}}}\right) .
$$

And using Lemma 2.4, we obtain the estimates of $\theta$. It is more simple for $\theta$ here, as the absence of the operator $\mathbb{P}$, but the estimates are still true, which are

$$
\begin{aligned}
& \|\theta(t)\|_{\dot{B}_{p, \infty}^{-1+\frac{n}{p}}} \leq C\left\|\theta_{0}\right\|_{\dot{B}_{p, \infty}^{-1+\frac{n}{p}}}+C \sup _{0<s<t} s^{\frac{\alpha}{2}}\|u(s) \otimes \theta(s)\|_{\dot{B}_{p, \infty}^{\alpha-2+\frac{n}{p}}}, \\
& t^{\frac{\alpha}{2}}\|\theta(t)\|_{\dot{B}_{p, \infty}^{\alpha-1+\frac{n}{p}}} \leq C\left\|\theta_{0}\right\|_{\dot{B}_{p, \infty}^{-1+\frac{n}{p}}}+C \sup _{0<s<t} s^{\frac{\alpha}{2}}\|u(s) \otimes \theta(s)\|_{\dot{B}_{p, \infty}^{\alpha-2+\frac{n}{p}}} .
\end{aligned}
$$

Summing (3.3) and (3.4) up, we have

$$
\begin{aligned}
\|\bar{M}(\theta)\|_{\Theta} & \leq C\left\|\theta_{0}\right\|_{\dot{B}_{p, \infty}^{\alpha-1+\frac{n}{p}}}+C \sup _{0<s<t} s^{\frac{\alpha}{2}}\|u(s) \otimes \theta(s)\|_{\dot{B}_{p, \infty}^{\alpha-2+\frac{n}{p}}} \\
& \leq C\left\|\theta_{0}\right\|_{\dot{B}_{p, \infty}^{\alpha-1+\frac{n}{p}}}+C \sup _{0<s<t} s^{\frac{1}{2}}\|u(s)\|_{L^{\infty}} s^{\frac{(\alpha-1)}{2}}\|\theta(s)\|_{\dot{B}_{p, \infty}^{\alpha-2+\frac{n}{p}}} \\
& \leq C\left\|\theta_{0}\right\|_{\dot{B}_{p, \infty}^{\alpha-1+\frac{n}{p}}}+C^{\prime}\|u\|_{U}\|\theta\|_{\Theta} .
\end{aligned}
$$

Define two complete metric spaces by

$$
U_{\epsilon}=\left\{u \in U \mid\|u\|_{U} \leq \epsilon\right\}, \quad \Theta_{\epsilon}=\left\{\theta \in \Theta \mid\|\theta\|_{\Theta}+\sup _{s>0} s^{\frac{\alpha}{2}}\|\theta(s)\|_{\dot{B}_{p, \infty}^{\alpha-3+\frac{n}{p}}} \leq \epsilon\right\} .
$$

Suppose $\theta_{1}, \theta_{2} \in \Theta$, by (3.5), we have

$$
\begin{aligned}
& \|\bar{M} \theta\|_{\Theta} \leq C\left\|\theta_{0}\right\|_{\dot{B}_{p, \infty}^{-1+\frac{n}{p}}}+C^{\prime} \epsilon\|\theta\|_{\Theta}, \quad \text { for } u \in U_{\epsilon}, \\
& \left\|\bar{M} \theta_{1}-\bar{M} \theta_{2}\right\|_{\Theta} \leq C^{\prime} \epsilon\left\|\theta_{1}-\theta_{2}\right\|_{\Theta} .
\end{aligned}
$$

According to the contraction mapping principle, equation (2.1) admits a unique solution $\theta \in \Theta_{\epsilon}$, provided that $C\left\|\theta_{0}\right\|_{\dot{B}_{p, \infty}^{-1+\frac{n}{p}}} \leq \frac{\epsilon}{2}$ and $\epsilon>0$ is sufficiently small.

Remark 3.1 To show the boundedness of the term $\sup _{s>0} S^{\frac{\alpha}{2}}\|\theta\|_{\dot{B}_{p, \infty}^{\alpha-3+\frac{n}{p}}}$, we choose $f(s)=$ $u(s) \otimes \theta(s)$ in Lemma 2.6, and by Lemma 2.3, we have

$$
\|u(s) \otimes \theta(s)\|_{\dot{B}_{p, \infty}^{\alpha-4+\frac{n}{p}}} \leq C\|u(s)\|_{\dot{B}_{p, \infty}^{-1+\frac{n}{p}}}\|\theta(s)\|_{\dot{B}_{p, \infty}^{\alpha-3+\frac{n}{p}}}
$$

thus we get

$$
\sup _{s>0} s^{\frac{\alpha}{2}}\|\theta(s)\|_{\dot{B}_{p, \infty}^{\alpha-3+\frac{n}{p}}} \leq C \frac{\left\|\theta_{0}\right\|_{\dot{B}_{p, \infty}^{-3+\frac{n}{p}}}}{1-C^{\prime}\|u\|_{U}},
$$

that is to say, if $\|u\|_{U}$ is small enough, the term $\sup _{s>0} S^{\frac{\alpha}{2}}\|\theta(s)\|_{\dot{B}_{p, \infty}^{\alpha-3+\frac{n}{p}}}$ is bounded. 
Now we deal with the operator $M$. Choosing $f(s)=u(s) \otimes u(s)$ in Lemma 2.4 and $f(s)=$ $\theta(s) e_{n}$ in Lemma 2.5, respectively, and using (3.2), we have

$$
\begin{aligned}
\|u(t)\|_{\dot{B}_{p, \infty}^{-1+\frac{n}{p}}} \leq & C\left\|u_{0}\right\|_{\dot{B}_{p, \infty}^{-1+\frac{n}{p}}}+C \sup _{0<s<t} s^{\frac{\alpha}{2}}\|u(s) \otimes u(s)\|_{\dot{B}_{p, \infty}^{\alpha-2+\frac{n}{p}}} \\
& +C \sup _{0<s<t} s^{\frac{\alpha}{2}}\|\theta(s)\|_{\dot{B}_{p, \infty}^{\alpha-3+\frac{n}{p}}}, \\
t^{\frac{\alpha}{2}}\|u(t)\|_{\dot{B}_{p, \infty}^{\alpha-1+\frac{n}{p}} \leq} & C\left\|u_{0}\right\|_{\dot{B}_{p, \infty}^{-1+\frac{n}{p}}}+C \sup _{0<s<t} s^{\frac{\alpha}{2}}\|u(s) \otimes u(s)\|_{\dot{B}_{p, \infty}^{\alpha-2+\frac{n}{p}}} \\
& +C \sup _{0<s<t} s^{\frac{\alpha}{2}}\|\theta(s)\|_{\dot{B}_{p, \infty}^{\alpha-3+\frac{n}{p}} .}
\end{aligned}
$$

Adding (3.6) to (3.7), and by the definition of $U$, we have

$$
\begin{aligned}
\|M u\|_{U} \leq & C\left\|u_{0}\right\|_{\dot{B}_{p, \infty}^{-1+\frac{n}{p}}}+C \sup _{0<s<t} s^{\frac{\alpha}{2}}\|u(s) \otimes u(s)\|_{\dot{B}_{p, \infty}^{\alpha-2+\frac{n}{p}}} \\
& +C \sup _{0<s<t} s^{\frac{\alpha}{2}}\|\theta(s)\|_{\dot{B}_{p, \infty}^{\alpha-3+\frac{n}{p}}} \\
\leq & C\left\|u_{0}\right\|_{\dot{B}_{p, \infty}^{-1+\frac{n}{p}}}+C \sup _{0<s<t} s^{\frac{1}{2}}\|u(s)\|_{L^{\infty}} s^{(\alpha-1) / 2}\|u(s)\|_{\dot{B}_{p, \infty}^{\alpha-2+\frac{n}{p}}} \\
& +C \sup _{0<s<t} s^{\frac{\alpha}{2}}\|\theta(s)\|_{\dot{B}_{p, \infty}^{\alpha-3+\frac{n}{p}}} \\
\leq & C\left\|u_{0}\right\|_{\dot{B}_{p, \infty}^{-1+\frac{n}{p}}}+C\|u\|_{U}^{2}+C \sup _{0<s<t} s^{\frac{\alpha}{2}}\|\theta(s)\|_{\dot{B}_{p, \infty}^{\alpha-3+\frac{n}{p}}} .
\end{aligned}
$$

Due to Remark 3.1, we know $C \sup _{0<s<t} s^{\frac{\alpha}{2}}\|\theta(s)\|_{\dot{B}_{p, \infty}^{\alpha-3+\frac{n}{p}}}$ is bounded. Similarly, by (3.8), we get

$$
\|M u-M v\|_{U} \leq C\left(\|u\|_{U}+\|v\|_{U}\right)\|u-v\|_{U}, \quad \text { for } u, v \in U,
$$

that is, due to $\mathbb{P} M u(t)=M u(t)$, we obtain $\nabla \cdot M u(t)=0$. By the definition of $U_{\epsilon}$, the previous analysis shows that

$$
\begin{aligned}
& \|M u\|_{U} \leq C\left\|u_{0}\right\|_{\dot{B}_{p, \infty}^{-1+\frac{n}{p}}}+C\|u\|_{U}^{2}+C\|\theta\|_{\Theta} \\
& \quad \leq C\left\|u_{0}\right\|_{\dot{B}_{p, \infty}^{-1+\frac{n}{p}}}+\frac{C\left\|\theta_{0}\right\|_{\dot{B}_{p, \infty}^{-3+\frac{n}{p}}}}{1-C^{\prime}\|u\|_{U}}+C \epsilon\|u\|_{U}, \quad \text { for } u \in U_{\epsilon}, \\
& \|M u-M v\|_{U} \leq C \epsilon\|u-v\|_{U}, \quad \text { for } u, v \in U_{\epsilon} .
\end{aligned}
$$

Therefore, on the basis of the contraction mapping principle, equation (2.1) admits a unique solution $u \in U_{\epsilon}$, provided that $C\left\|u_{0}\right\|_{\dot{B}_{p, \infty}^{-1+\frac{n}{p}}} \in \frac{\epsilon}{3}, C\left\|\theta_{0}\right\|_{\dot{B}_{p, \infty}^{-3+\frac{n}{p}}} \in \frac{\epsilon}{3}$ and $\epsilon>0$ is sufficiently small.

The proof of Theorem 1.1 is done. 


\section{Publisher's Note}

Springer Nature remains neutral with regard to jurisdictional claims in published maps and institutional affiliations.

\section{Received: 23 September 2016 Accepted: 21 March 2017 Published online: 31 March 2017}

\section{References}

1. Majda, A: Introduction to PDEs and Waves for the Atmosphere and Ocean. Courant Lecture Notes in Mathematic, vol. 9. Am. Math. Soc., Providence (2003)

2. Pedlosky, J: Geophysical Fluid Dynamics. Springer, New York (1987)

3. Shahmurov, R, Hadji, L: Nonlinear stable steady solutions to the Ostroumov problem. Int. J. Heat Mass Transf. 82, 604-612 (2015)

4. Zhang, Z: Some regularity criteria for the 3D Boussinesq equations in the class $L^{2}\left(0, T ; \dot{B}_{\infty, \infty}^{-1}\right)$. ISRN Appl. Math. 2014, Article ID 564-758 (2014). doi:10.1155/2014/564758

5. Xu, F, Zhang, Q, Zheng, X: Regularity criteria of the $3 \mathrm{D}$ Boussinesq equations in the Morrey-Campanato space. Acta Appl. Math. 121, 231-240 (2012)

6. Fan, J, Zhou, Y: A note on regularity criterion for the 3D Boussinesq system with partial viscosity. Appl. Math. Lett. 22(5), 802-805 (2009)

7. Mechdene, M, Gala, S, Guo, ZG, Ragusa, AM: Logarithmical regularity criterion of the three-dimensional Boussinesq equations in terms of the pressure. Z. Angew. Math. Phys. 67(5), 67-120 (2016). doi:10.1007/s00033-016-0715-2

8. Jia, $Y, Z$ Zhang, $X$, Dong, B: Logarithmical regularity criteria of the three-dimensional micropolar fluid equations in terms of the pressure. Abstr. Appl. Anal. 2012, Article ID 395420 (2012). doi:10.1155/2012/395420

9. Zhang, Z: A logarithmically improved regularity criterion for the 3D Boussinesq equations via the pressure. Acta Appl. Math. 131, 213-219 (2014)

10. Yao, ZA, Wang, Q, Bie, Q: On the well-posedness of the inviscid Boussinesq equations in the Besov-Morrey spaces. Kinet. Relat. Models 8(3), 395-411 (2015)

11. Liu, X, Wang, M, Zhang, Z: Local well-posedness and blowup criterion of the Boussinesq equations in critical Besov spaces. J. Math. Fluid Mech. 12, 280-292 (2010). doi:10.1007/s00021-008-0286-X

12. Jiu, Q, Miao, C, Wu, J, Zhang, Z: The $2 D$ incompressible Boussinesq equations with general critical dissipation. SIAM J. Math. Anal. 46, 3426-3454 (2014)

13. Stefanov, A, Wu, J: A global regularity result for the 2D Boussinesq equations with critical dissipation. J. Anal. Math., accepted for publication; arXiv:1411.1362v3 [math.AP]

14. $\mathrm{Wu}, J, \mathrm{Xu}, \mathrm{X}, \mathrm{Xue}, \mathrm{L}, \mathrm{Ye}, \mathrm{Z}$ : Regularity results for the $2 \mathrm{D}$ Boussinesq equations with critical and supercritical dissipation. Commun. Math. Sci. 14, 1963-1997 (2016)

15. Ye, Z: Global smooth solution to the 2D Boussinesq equations with fractional dissipation. arXiv:1510.03237v2 [math.AP]

16. Ye, Z, Xu, X: Global well-posedness of the 2D Boussinesq equations with fractional Laplacian dissipation. J. Differ. Equ. $260,6716-6744(2016)$

17. Abidi, H, Hmidi, T: On the global well-posedness for Boussinesq system. J. Differ. Equ. 233(1), 199-220 (2007)

18. Hou, T, Li, C: Global well-posedness of the viscous Boussinesq equations. Discrete Contin. Dyn. Syst. 12(1), 1-12 (2005)

19. Ye, Z: Blow-up criterion of smooth solutions for the Boussinesq equations. Nonlinear Anal. 110, 97-103 (2014)

20. Ye, Z: Regularity criteria for 3D Boussinesq equations with zero thermal diffusion. Electron. J. Differ. Equ. 2015, 97 (2015)

21. Ye, Z: A logarithmically improved regularity criterion of smooth solutions for the 3D Boussinesq equations. Osaka J. Math. 53, 417-423 (2016)

22. Yang, X, Qin, Y: A Beale-Kato-Majda criterion for the 3D viscous magnetohydrodynamic equations. Math. Methods Appl. Sci. 38(4), 701-707 (2015)

23. Yang, X, Zhang, L: BKM's criterion of weak solutions for the 3D Boussinesq equations. J. Partial Differ. Equ. 27(1), 64-73 (2014)

24. Tian, C, Yang, X: A Beale-Kato-Majda regularity criteria to the $2 \mathrm{D}$ viscous $M H D$ equations in BMO space. Int. J. Contemp. Math. Sci. 8(1-4), 117-123 (2013)

25. Gala, S, Guo, Z, Ragusa, A: A remark on the regularity criterion of Boussinesq equations with zero heat conductivity. Appl. Math. Lett. 27, 70-73 (2014)

26. Qin, Y, Yang, X, Wang, Y, Liu, X: Blow-up criteria of smooth solutions to the 3D Boussinesq equations. Math. Methods Appl. Sci. 35(3), 278-285 (2012)

27. Cui, X, Dou, C, Jiu, Q: Local well-posedness and blow up criterion for the inviscid Boussinesq system in Hölder spaces. J. Partial Differ. Equ. 25(3), 220-238 (2012)

28. Chen, ZM, Xin, ZP: Homogeneity criterion for the Navier-Stokes equations in the whole spaces. J. Math. Fluid Mech. 3 , $152-182(2001)$

29. Triebel, H: Theory of Functions Spaces II. Birkhäuser, Basel (1992)

30. Miao, C, Yuan, B, Zhang, B: Well-posedness of the Cauchy problem for the fractional power dissipative equations. Nonlinear Anal. 68, 461-484 (2008)

31. Frazier, M, Jawerth, B, Weiss, G: Littlewood-Paley Theory and the Study of Function Spaces. Am. Math. Soc., New Jork (1991)

32. Bergh, J, Löfström, J: Interpolation Spaces: An Introduction. Grundle der Mathematischen Wissenschaften, vol. 233. Springer, Berlin (1976) 